\title{
First Fenestrated Endovascular Aortic Aneurysm Repair in Pakistan
}

\author{
Usama Khalid Choudry ${ }^{1}$, Omer Ehsan ${ }^{2}$, Zahid Amin Khan ${ }^{3}$ and Amina Amin ${ }^{1}$ \\ ${ }^{1}$ Department of General Surgery, Shifa International Hospital, Islamabad, Pakistan \\ ${ }^{2}$ Department of Vascular Surgery, Shifa International Hospital, Islamabad, Pakistan \\ ${ }^{3}$ Department of Interventional Radiology, Shifa International Hospital, Islamabad, Pakistan
}

\begin{abstract}
Fenestrated grafts form an alternative to open surgery, and are already increasingly being offered as part of the standard spectrum of treatment in developed countries. The past two decades have seen rapid developments in fenestrated endovascular aneurysm repair (fEVAR)/branched (bEVAR) expertise, to the point where the results are striking and can compete with those of conventional surgery. We here report the case of an 82-year male, who was diagnosed with an abdominal aortic aneurysm 10 years ago. Computed tomography showed a large fusiform juxta-renal abdominal aortic aneurysm extending into bilateral common iliac arteries to their bifurcation. Bilateral femoral access was acquired surgically. Fenestrated part of the stent grafted was deployed successfully. Right and left renal arteries and superior mesenteric artery were stented. Celiac artery showed good flow and no stenting was required. Distal stent graft and bilateral iliac grafts were deployed successfully. Follow up computed tomography scan showed $99 \%$ patency and no endoleak. Patient remains stable and asymptomatic at 6-month follow-up. To the best of our knowledge, it is the first reported case of successful fEVAR from Pakistan.
\end{abstract}

Key Words: Fenestrated grafts, Abdominal aortic aneurysm, Endovascular.

How to cite this article: Choudry UK, Ehsan O, Khan ZA, Amin A. First Fenestrated Endovascular Aortic Aneurysm Repair in Pakistan. J Coll Physicians Surg Pak 2021; 31(04):469-471.

\section{INTRODUCTION}

The management of a juxta-renal aortic aneurysm (AA) by fenestrated endovascular aneurysm repair (fEVAR) was first done in 1999. ${ }^{1}$ fEVAR has become the most widely employed treatment modality for juxta-renal aneurysms, with 263 cases reported in United Kingdom in 2015. ${ }^{2}$ The UK-wide BSET GLOBALSTAR (British Society for Endovascular Therapy: Global Collaborators on Advanced Stent-Graft Techniques for Aneurysm Repair) registry has recognised the safety and effectiveness of the procedure by reporting short- and medium-term outcomes of the preliminary procedures performed during $2003-2009 .{ }^{3}$ However, reports of long-term outcomes from Cleveland Clinic on long-term durability show a $38 \%$ risk of component separation after 1 year follow-up. ${ }^{4}$

Fenestrated and branched (bEVAR) grafts form an alternative to open surgery, and are already increasingly being preferred as part of the standard spectrum of treatment in major aortic centres.

Correspondence to: Dr. Omer Ehsan, Department of Vascular Surgery, Shifa International Hospital, Islamabad, Pakistan

E-mail:dromerehsan@gmail.com

Received: August 05, 2019; Revised: August 05, 2019;

Accepted: October 01, 2019

DOI: https://doi.org/10.29271/jcpsp.2021.04.469
The past 20 years have seen rapid advances in fEVAR/bEVAR technology, to the point where the results are striking and can compete with those of conventional surgery. ${ }^{5}$ There is a considerable debate about the impact of fEVAR in terms of long-term cost effectiveness compared to open surgical repair. ${ }^{6}$ The cost of fEVAR is owed to the need for customisation of the graft. While this procedure may be successful in developed countries, its feasibility in the developing nations is a question unanswered yet.

We, hereby, report the first case of successful application of fEVAR in Pakistan, a developing nation in the South Asian belt.

\section{CASE REPORT}

An 82-year healthy male presented to the surgical OPD with an incidentally discovered abdominal AA, 10 years ago. Computed tomography (CT) showed a large fusiform juxta-renal abdominal AA, measuring approximately $56 \times 58 \times 93 \mathrm{~mm}$. The aneurysmal portion was extending into bilateral common iliac arteries till their bifurcation. The diameter of aorta at renal arteries was $1.8 \mathrm{~cm}$. Neck was conical and the diameter increased to $3.1 \mathrm{~cm}$ at $1.5 \mathrm{~cm}$ below the renal arteries (Figure 1). The prospect of fenestrated endovascular stent graft was discussed with the patient and his family. Patient was kept on optimum blood pressure control, while the customised graft was being made overseas. Procedure was performed under 
general anesthesia. Bilateral femoral access was acquired surgically. Left brachial artery was percutaneously accessed for pigtail catheter, which was advanced into thoracic aorta. Fenestrated part of the stent grafted was deployed successfully. Right and left renal arteries and superior mesenteric artery were stented. Celiac artery showed good flow and no stent was required. Distal abdominal stent graft and bilateral iliac grafts were deployed successfully (Figure 2). Postoperatively, patient remained stable and showed no signs of spinal ischemia, cerebrovascular accident or renal failure. Follow-up CT scan showed complete patency and no endoleak (Figure 3). Patient remained stable and asymptomatic at six months follow-up.

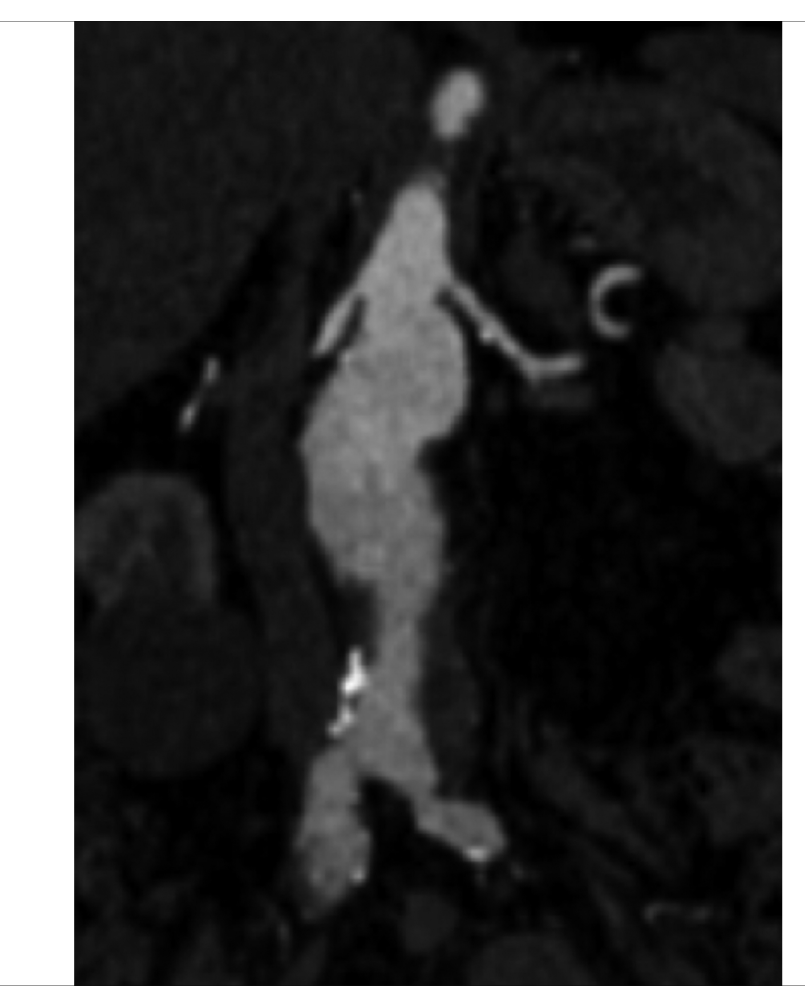

Figure 1: Preoperative computed tomography angiography (CTA) of the patient's aneurysm.

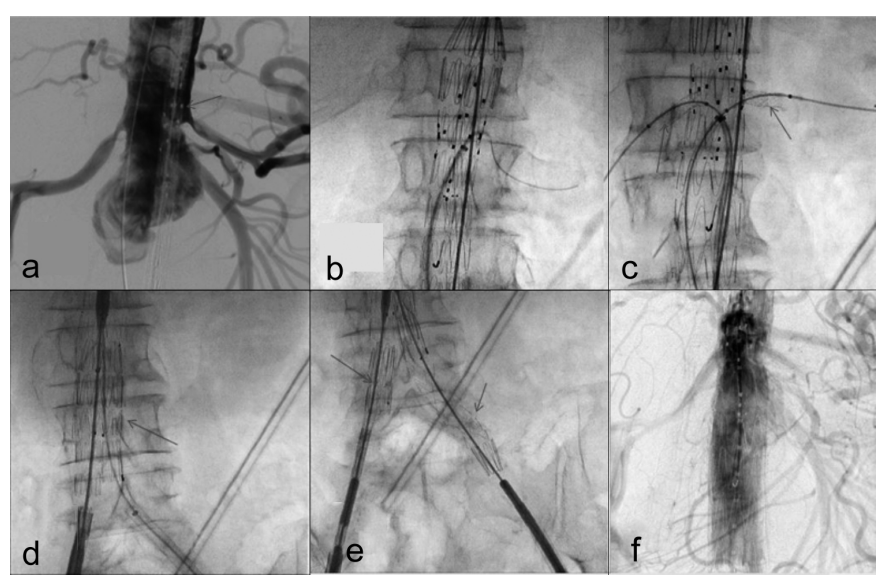

Figure 2: (a) Showing fenestrated graft at aneurysm site (red arrow), (b) showing deployed fenestrated graft, (c) showing stented right and left renal arteries (red arrows), (d) showing abdominal portion of stent graft, (e) showing bilateral iliac stent grafts in place, (f) showing final assembly of fEVAR.

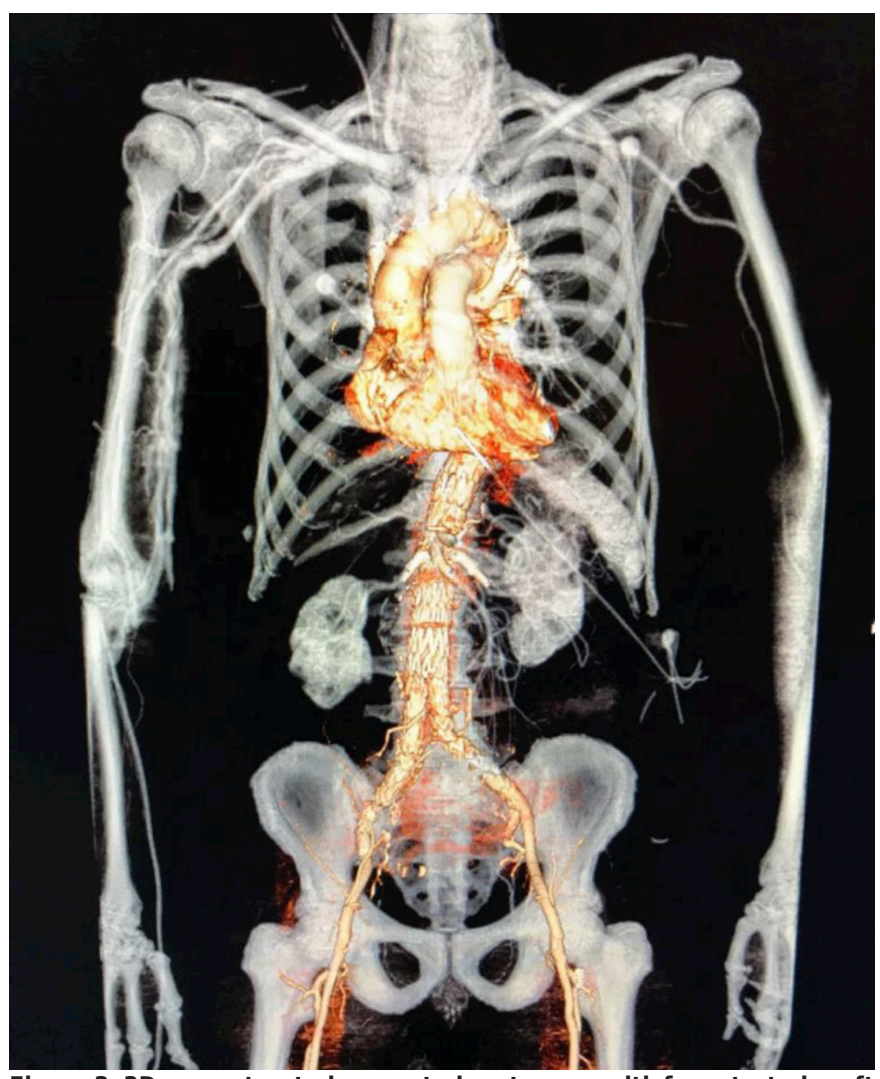

Figure 3: 3D reconstructed computed aortogram with fenestrated graft in place.

\section{DISCUSSION}

Successful deployment of fEVAR depends on many factors. A high degree of technical expertise in vascular surgical and interventional techniques is important to reduce complications. The first successful fEVAR was performed in $1996 .{ }^{7}$ However, its advent in the developing world has been more or less non-existent. The mandatory requirement forthis procedure is establishment of a centre that has both interventional and open aortic surgery facilities. Endovascular aortic surgery as an emerging modality was expected to reduce the health expenditure due to short hospital stays and less complications, but the reduction outcasts the high device price deployed in the procedure. ${ }^{6}$ However, cost expenditure and analysis depend on multiple factors. Patient opting for fEVAR/bEVAR has to wait for custom-made devices, which may lead to increase in waiting time, ultimately contributing to non-modifiable time-dependent patient mortality. ${ }^{8}$

A recent meta-analysis comparing fEVAR with open surgical repair (OSR) recognised 35 case series with data on 2,326 patients. Perioperative mortality was found to be similar at $4.1 \%$ in both open repair and fEVAR case series (odds ratio $[O R]=1.059, p=0.822)$. Postoperative renal insufficiency was also not significantly different $(O R=1.136, p=0.542)$. Yet, fEVAR patients had higher rates of secondary re-intervention, renal impairment during follow-up, and a lower long-term survival compared with open repair patients. ${ }^{9}$

The chances of conversion to OSR due to complications, such as 
endo-leak and graft migration and re-intervention, add to the cost. Other complications, such as cardiac, pulmonary, neurological, and renal complications are included too. Furthermore, patient who has suffered from complications, such as disabling stroke, generates more cost with low quality of life than rest of the population.

On a long run, these costs include readmission after the initial admission. The exposure to radiation for follow-up CT scans and fluoroscopy during intervention is one of the factors for cost increment. $^{10}$

There is a need for establishment of centres with technical expertise and funding in order to change the standard of care for abdominal AAs' repair in the developing world.

\section{PATIENT'S CONSENT:}

Informed consent was obtained from the patient to publish this case data.

\section{CONFLICT OF INTEREST:}

The authors declared no conflict of interest.

\section{AUTHORS' CONTIBUTION:}

UKC, AA: Put the case report together and wrote it up.

OE, ZAK: Reviewed the script.

\section{REFERENCES}

1. Browne TF, Hartley D, Purchas S, Rosenberg M, Van Schie G, Lawrence-Brown M. A fenestrated covered suprarenal aortic stent. Eur J Vasc Endovasc Surg 1999; 18:445-9. doi: 10.1053/ejvs.1999.0924.

2. Waton S, Johal A, Heikkila K, Cromwell D, Loftus I. National vascular registry: 2015 Annual Report. Royal College of Surgeons of England. London 2015.

3. British society for endovascular therapy and the global collaborators on advanced stent-graft techniques for aneurysm repair (Globalstar) registry. Early results of fenestrated endovascular repair of juxtarenal aortic aneurysms in the United Kingdom. Circulation 2012; 125:2707-2715. doi: 10.1161/CIRCULATIONAHA.111.070334.

4. Eagleton MJ. FEVAR: Long-term data from the Cleveland clinic. Endovasc Today 2017; 16:5-7.

5. Anderson JL, Adam DJ, Berce M, Hartley DE. Repair of thoracoabdominal aortic aneurysms with fenestrated and branched endovascular stent grafts. J Vasc Surg 2005; 42(4):600-7. doi: 10.1016/j.jvs.2005.05.063.

6. Takayama Y. A Cost-utility analysis of endovascular aneurysm repair for abdominal aortic aneurysm. Ann Vasc Dis 2017; 10(3):185-91. doi: 10.3400/avd.oa.17-00059.

7. Park JH, Chung JW, Choo IW. Fenestrated stent-grafts for preserving visceral arterial branches in the treatment of abdominal aortic aneurysms: Preliminary experience. J VascIntervRadiol 1996; 7:819-823. doi: 10.1016/s1051-0443(96)70854-0.

8. Armstrona N. Buraers L. Deshnande S. Al M. Riemsma R. Vallabhaneni SR, et al. The use of fenestrated and branched endovascular aneurysm repair for juxtarenal and thoracoabdominal aneurysms: A systematic review and cost-effectiveness analysis. Health Technol Assess 2014; 18(70): 1-66. doi: 10.3310/hta18700.

9. Rao R, Lane TR, Franklin IJ, Davies AH. Open repair versus fenestrated endovascular aneurysm repair of juxtarenal aneurysms. J Vasc Surg 2015; 61(1):242-55 doi: 10.1016/j.jvs.2014.08.068.

10. Chambers D, Epstein D, Walker S, Fayter D, Paton F, Wright $\mathrm{K}$, et al. Endovascular stents for abdominal aortic aneurysms: A systematic review and economic model. Health Technol Assess 2009; 13(48) doi: $10.3310 /$ hta13480. 